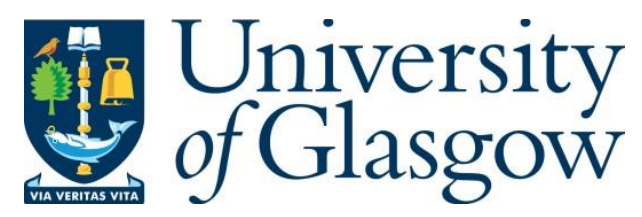

Flynt, A. and Dean, N. (2016) A survey of popular R packages for cluster analysis. Journal of Educational and Behavioral Statistics, 41(2), pp. 205-225.

There may be differences between this version and the published version. You are advised to consult the publisher's version if you wish to cite from it.

http://eprints.gla.ac.uk/153580/

Deposited on: 15 December 2017

Enlighten - Research publications by members of the University of Glasgow http://eprints.gla.ac.uk 


\title{
A Survey of Popular R Packages for Cluster Analysis
}

\author{
January 8, 2017
}

\begin{abstract}
Cluster analysis is a set of statistical methods for discovering new group/class structure when exploring datasets. This article reviews the following popular libraries/commands in the $\mathrm{R}$ software language for applying different types of cluster analysis: from the stats library, the kmeans and hclust functions; the mclust library; the poLCA library; and the clustMD library. The packages/functions cover a variety of cluster analysis methods for continuous data, categorical data or a collection of the two. The contrasting methods in the different packages are briefly introduced and basic usage of the functions is discussed. The use of the different methods is compared and contrasted and then illustrated on example data. In the discussion, links to information on other available libraries for different clustering methods and extensions beyond basic clustering methods are given. The code for the worked examples in Section 2 is available at http://www.stats.gla.ac.uk/ ^nd29c/Software/ClusterReviewCode.R.
\end{abstract}

Keywords: Cluster Analysis; R software language; K-means; Hierarchical Clustering; ModelBased Clustering 


\section{Introduction}

Cluster analysis is an area of statistics involved with finding groups in data by aggregating items that are similar in some sense into separate classes (Everitt et al., 2011). Any field that has interest in finding meaningful groups within data could benefit from statistical clustering. Some recent applications have included using clustering to group student skill set profiles (Dean and Nugent, 2013), to group rates of obesity and diabetes in US counties (Flynt and Daepp, 2015), and to estimate the spatial patterns in disease risk (Anderson et al., 2014).

This article discusses a selection of libraries and commands in the $\mathrm{R}$ software language ( $\mathrm{R}$ Core Team, 2015) for implementing some of the most popular methods for algorithmic and parametric clustering. The structure of the remainder of this article is as follows: in Section 2 we briefly review the models/algorithms for k-means and hierarchical clustering (2.2), model-based clustering and latent class analysis (2.3) and introduce the package/commands for implementing these methods. We apply the different packages to example data and review the relevant output before concluding with a discussion in Section 3 and pointers towards other packages for further cluster analysis extensions. Note that R commands and libraries will be indicated with this font, while the command arguments will be indicated with an alternative font.

\section{Common Cluster Analysis Methods and their Impementation}

Given that data are clustered such that the objects within groups found are similar in some way(s), there are obviously different methods for defining similarity. For example, one of the most popular methods for defining dissimilarity is using Euclidean distance from the cluster center which leads to the k-means method, or using the density of points in the surrounding area which leads to model-based clustering. The many different definitions of (dis)similarity have led to a wide variety of clustering methodologies. These can be roughly divided into three main categories: algorithmic, parametric and 
non-parametric. Some of the oldest cluster analysis methods such as k-means (MacQueen, 1967) and hierarchical clustering (Ward, 1963) are examples of the algorithmic class of methods. Methods such as model-based clustering (Wolfe, 1963; Fraley and Raftery, 2002) and latent class analysis (Lazarsfeld, 1950a,b; Lazarsfeld and Henry, 1968), based on finite mixture models (McLachlan and Peel, 2000), are examples of parametric clustering. Non-parametric clustering (mentioned again in Section 3) looks to identify modes in the estimated density surface for the data with clusters.

We now introduce the simulated dataset used to illustrate the cluster analysis methods described and implemented in the remaining subsections. Code and selected output will be presented that can be adapted for the reader's own data analysis.

\subsection{Data}

To illustrate the discussed $\mathrm{R}$ packages, we will present results from a simulated dataset of 600 observations with two continuous variables and 4 discrete ( 3 binary, 1 ternary) variables. The continuous variables, named vars, create 3 underlying groups, two of which are spherical and one elliptical. These simulated groups can be seen in Figure 1a (177 observations in black, 237 in red, 186 in green). The binary variables, named c1, c2, c3, c4, were simulated in such a way that one variable had uniform probability of success across the groups, another had higher success probability for the black group and the final had higher success probability for the red group. In the ternary variable, there was a higher probability of being assigned a 1 in the black group, a 2 in the red group and a 3 in the green group. The exact distribution of these variables within each underlying group can be seen in Figure 1b. Throughout the remainder of this section, the estimated clustering from each method will be compared to the true simulated groups, named by their color in Figure 1a.

In the following sections, $\mathrm{R}$ code to be implemented is prefaced by the prompt symbol $>$ (which would be omitted if copying this into an $\mathrm{R}$ console), comments and output not meant to be implemented is prefaced by \#. 


\subsection{Algorithmic Clustering Methods}

\subsubsection{K-means}

K-means is based on the idea that points should be assigned to the clusters that minimize the overall distance between points and the cluster means/centroids to which they have been assigned. The typical distance measure used is Euclidean (although other distances can theoretically be used), so the method is usually only applied to continuous data. K-means assumes the variability in all clusters in all variables is the same, resulting in the discovery of spherical clusters which each take up an equal volume in variable space. If variables are measured on vastly different scales, it is a good idea to scale them prior to applying k-means; the $\mathrm{R}$ command scale can be used to automatically center and/or scale vectors. The most common command for implementing $\mathrm{k}$-means in $\mathrm{R}$ is the kmeans command located in the stats library.

One of the issues in any clustering method is the decision as to how many clusters are present in the data being examined. The most common way to choose the number of clusters for k-means is by fitting k-means models for a range of consecutive numbers, usually 1 up to some maximum number, and plotting an elbow plot of the total within sum of squares value for each number of clusters versus that cluster number. An example is given in Figure 2 in Section 2.2.2. The number of clusters is taken to be the 'elbow' or turning point in the line plot where the reduction in sum of squares seems to drop off. The assignment of points into clusters for a particular model is obtained by extracting the cluster assignment, cluster, in the resulting fitted object list.

Other non-visual methods for choosing the number of clusters (for any clustering method, not just k-means) are performed by calculating some metric that measures goodness of aspects of the clustering. Examples of these measures, found in the fpc library (Hennig, 2015) are: average silhouette width, the Calinski and Harabasz index and a Pearson version of Hubert's gamma coefficient. 


\subsubsection{Simulated Data Results and Code for K-Means}

The first argument in the kmeans command is the data matrix (rows=observations, columns=variables), then the second argument centers can either be set to the number of clusters required or a matrix of data points representing the initial cluster centers. The default value for the argument nstart which specifies how many times the algorithm is run with random starts before choosing the best result is 1 , which is not to be advised. A larger value, at least 10 or more if time allows, particularly as the dimensionality/number of variables rises, is to be recommended.

Here we run k-means for different values of $\mathrm{k}$, from 1 to 9 , with 50 random starts on the first two columns/continuous variables in our simulated dataset vars.

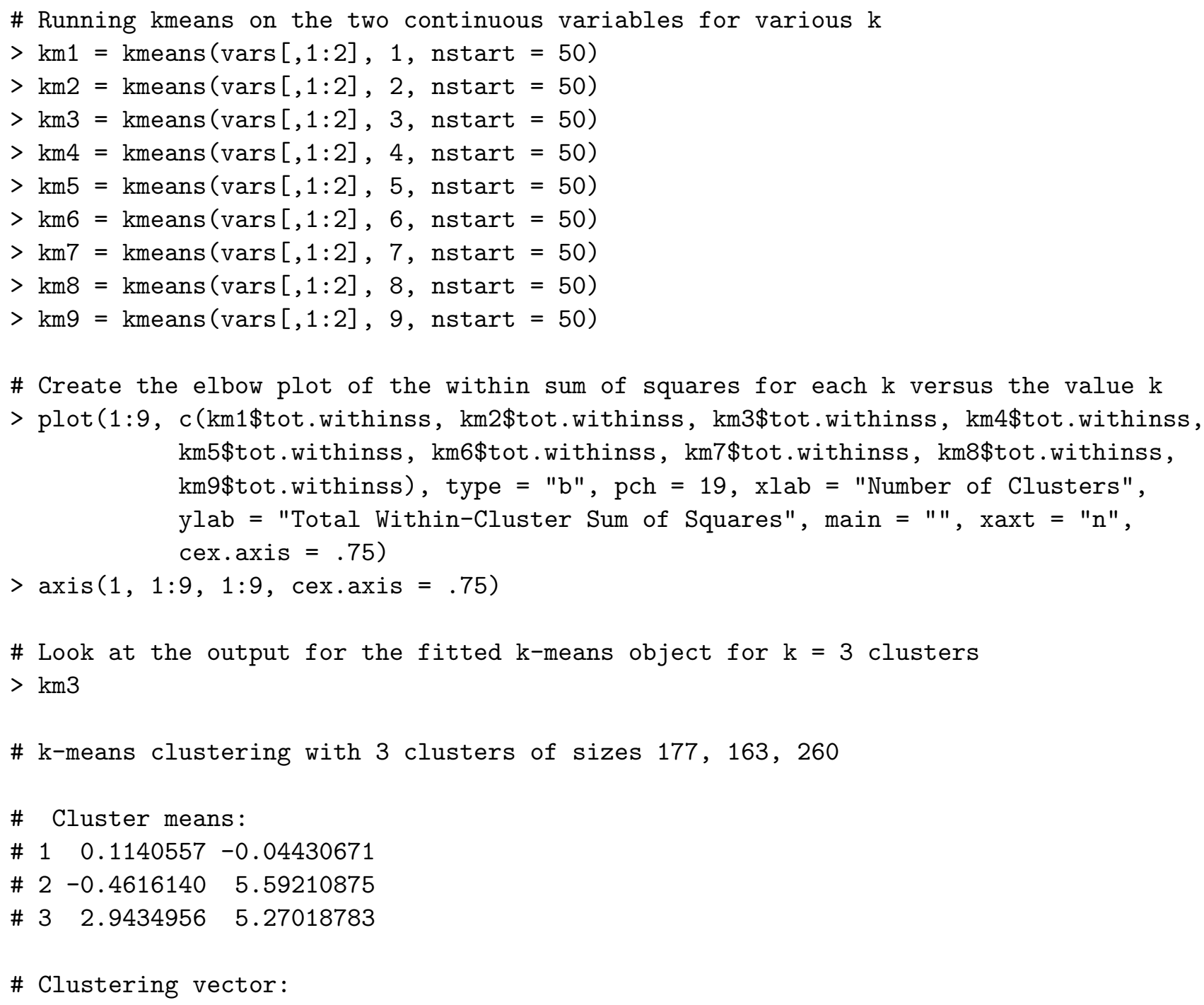




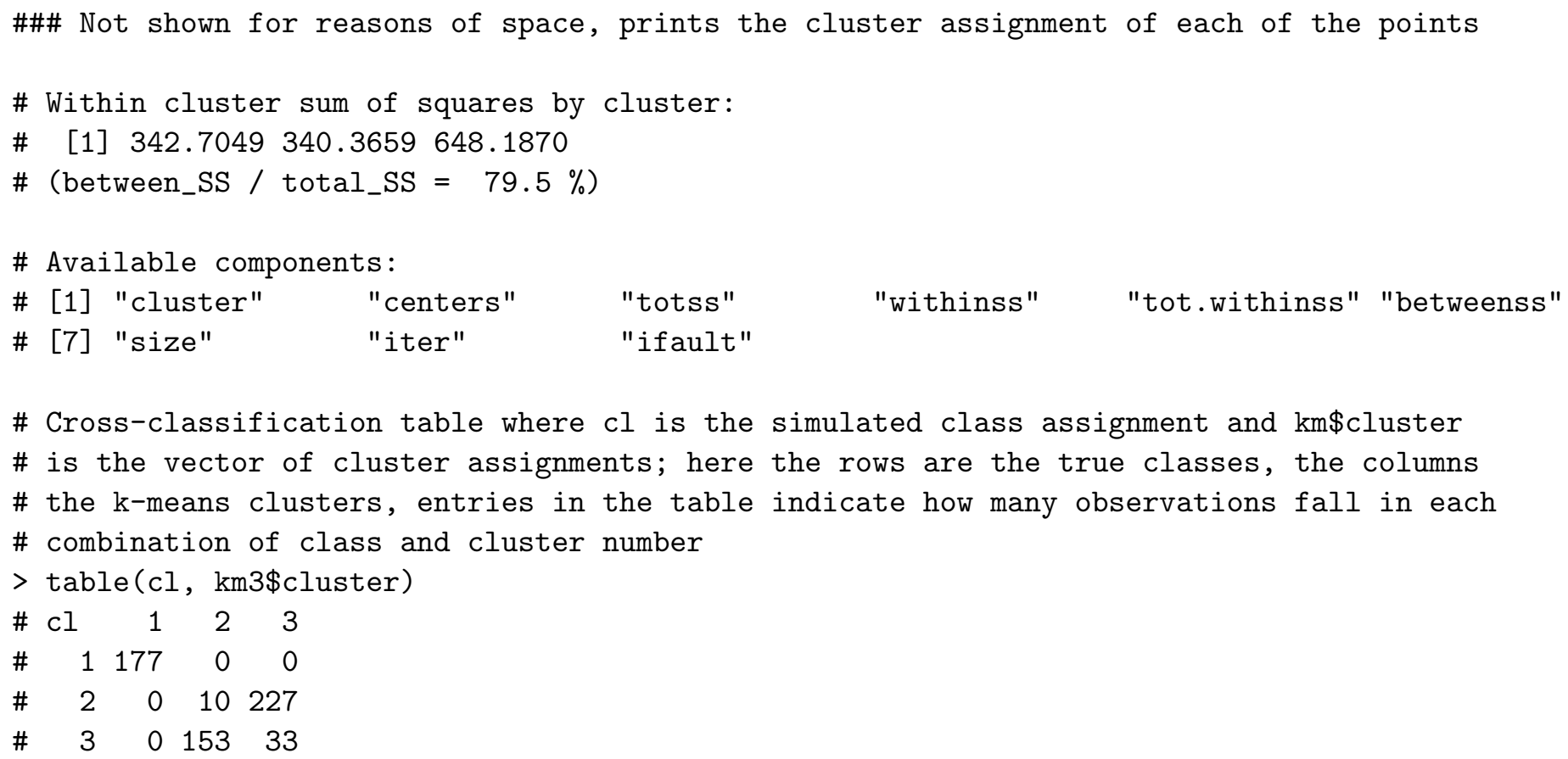

The elbow plot from the resulting k-means models can be seen in Figure 2. This plot suggests the choice of the 3 cluster model. It is common to create a cross-classification table (see table in output) comparing the true simulated groups and the estimated clusters. Note that the estimated clusters may have labels switched from the true groups (as seen above), but can be reordered appropriately. The misclassification rate is calculated as the number of misclassified observations out of the total number of observations. We see that for the 3 cluster solution produced by k-means, the black group was perfectly recovered, but 43 observations were misclassified in the overlapping red and green groups, resulting in a misclassication rate of $42 / 600=0.071 \overline{6}$.

\subsubsection{Hierarchical Agglomerative Clustering}

Hierarchical agglomerative clustering is based on the idea of successively merging the pair of closest/most similar clusters together to form a new larger cluster until all points are merged into one cluster. Usually, the order and height of merges is visualized in a tree-like diagram called a dendrogram (sometimes dendogram); see Figure 3 in Section 2.2.4 for an example. The advantage of this method is the ability to easily visualize the structure of the data regardless of how high-dimensional it may 
be. This method of clustering also allows for hierarchy in the clusters found.

There are several ways to calculate linkages, the distance between pairs of non-singelton clusters. Each definition of linkage gives rise to a different type of clustering solution on the same data. Single linkage defines the distance between two clusters as the minimum of all the pairwise distances between pairs of points in the different clusters. This type of linkage give rise to 'chaining', where the merges are more likely to have points or small clusters joining with a large cluster rather than forming new large clusters. This effect can lead to groups that are of unusual shape (e.g. non-spherical, non-elliptical) but can also lead to close groups being incorrectly merged if there is any degree of overlap. Single linkage is usually very useful in identifying outliers. This linkage is also related to the minimal spanning tree (see Gower and Ross (1969) for details). Complete linkage is the maximum of the pairwise distances between all pairs of points in the different clusters. It tends to separate out overlapping groups well, but will do poorly recovering non-standard shapes of groups. Average linkage uses the average pairwise distance between all pairs of points in the different clusters as the measure of distance. The clustering for average linkage usually lies somewhere between the solutions given by single and complete linkage. Centroid linkage defines distance between clusters as the distance between the cluster centroids. Ward's linkage looks at the increase in sum of squared deviations from cluster mean before and after merge as the measure of similarity. This linkage tends to find compact spherical clusters similar to k-means clustering.

\subsubsection{Simulated Data Results and Code for Hierarchical Clustering}

Hierarchical agglomerative clustering in $\mathrm{R}$ is generally carried out using the hclust command in the stats library.

The first argument is a distance object, containing all the pairwise distances between points in the dataset. This can be created using the dist function. If the dist function is not used, then the as.dist function may be needed to force the object into the correct format. The next argument is 
the linkage method (for example, method = "complete"). The plot command plots a dendrogram and a colored dendrogram can be produced using the ColorDendrogram function in the sparcl library (Figure 3). The command cutree is used to classify the data points into the clusters where the fitted dendrogram is cut with either the number of clusters or the height at which the tree should be cut.

Figure 3 shows a colored dendrogram of the results from hierarchical clustering with average linkage. If we choose to cut the tree at 3 clusters, the black group is completely recovered, but the green group is almost entirely merged into the red group, resulting in a misclassification rate of 0.30 .

The code for implementing this is given below.

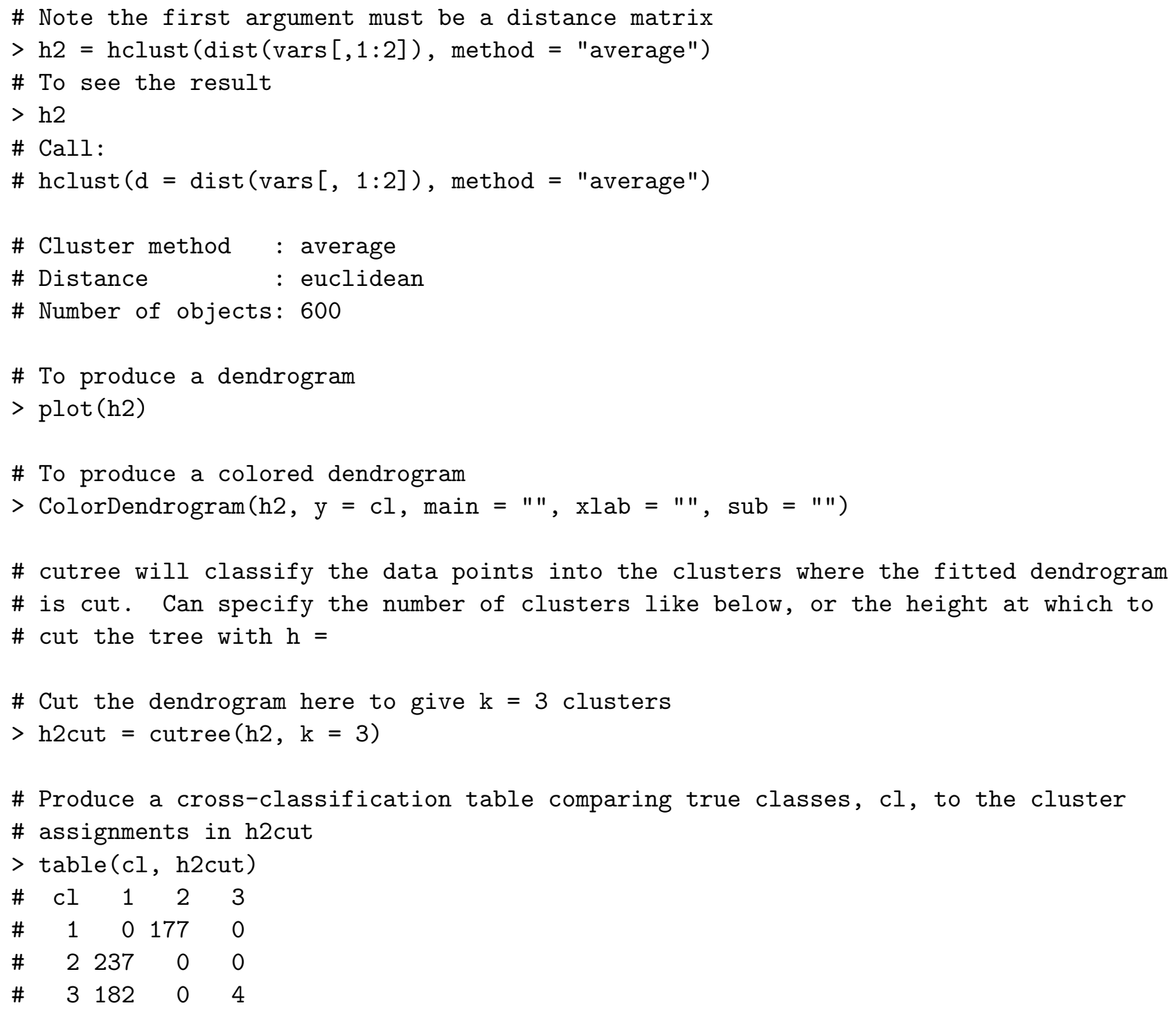




\subsection{Clustering Methods Based on Finite Mixture Models}

Model-based clustering, a parametric method, is based on estimating a statistical model rather than optimizing a function. The model on which model-based clustering and latent class analysis is based is the finite mixture model (McLachlan and Peel, 2000). Instead of modeling the data as a single population with a single distribution, the finite mixture model assumes that the population is made up of multiple sub-populations. Each of the sub-populations has an associated weight equal to its associated proportion in the whole population, as well as a component-specific distribution that models the data in the sub-population. The density for the data is then given by the following equation:

$$
f(\mathbf{x})=\sum_{k=1}^{K} \tau_{k} f_{k}(\mathbf{x}), \quad 0 \leq \tau_{k} \leq 1, \quad \sum_{k=1}^{K} \tau_{k}=1
$$

Here $\tau_{k}$ is the $k^{t h}$ mixing proportion equal to the proportion this component sub-population makes up of the whole population and $f_{k}()$ is the $k^{t h}$ component density. Usually, the component densities all come from the same parametric family. The EM algorithm (Dempster et al., 1977) is used to estimate the model parameters. It is common for each component to be associated with a cluster. The probability of belonging to each component is calculated for each observation and the observation can be assigned to the component/cluster for which it has largest probability of membership.

Often, the number of components that best fits the data is equivalent to the number of clusters present in the data, so that choosing the number of clusters becomes a model-choice problem, with different numbers of clusters/components defining a different model. The most common way to identify the best fitting number of components is to fit different models for a range of numbers of clusters, score each model and select the model with the best score. The most common scoring measure used is the Bayesian Information Criterion (BIC) (Schwarz, 1978). 


\subsubsection{Model-Based Clustering}

Model-based clustering $(\mathrm{mbc})$ is the common name for finite mixture model clustering for continuous data. In model-based clustering, the component density $f_{k}$ is Gaussian with corresponding mean and variance estimates. That is, each component can be summarized by a mean, variance and a mixing proportion.

\subsubsection{Simulated Data Results and Code for Model-Based Clustering}

In mbc, the unrestricted variance matrix for component $k$ is high dimensional and difficult to estimate, so the mclust package, fits different variance matrix parameterizations. For univariate data the options are 'E' or 'V' (equal or variable across components) and a variety of different options exist for multivariate data indexed by three letters 'XXX', the first letter indicating the volume of the components, either 'E' or ' $\mathrm{V}$ '; the second letter indicating the shape of the components, either ' $\mathrm{E}$ ' or ' $\mathrm{V}$ ' or 'I' (for the identity matrix) and the third letter indicating the orientation of the components, either 'E', 'V' or 'I'. K-means is extremely similar to the model with either 'E' for univariate or 'EII' for multivariate data. In the mclust library (Fraley et al., 2012) the function Mclust is used to fit the finite mixture model.

The first argument for the Mclust command is the data matrix, the second is the range of number of components to be fit (default is 1 to 9 ), and the best model chosen is the one with the highest BIC value. The identified best model (in terms of variance parameterization and number of components, see Figure 4a) can be found by using the summary command on the fitted Mclust object. The classification element of the fitted mclust object gives the assignment of data points to clusters.

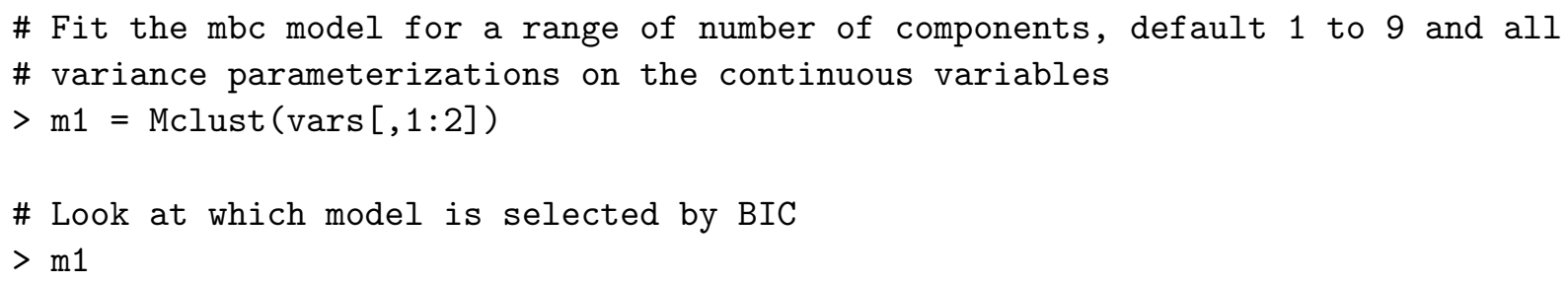




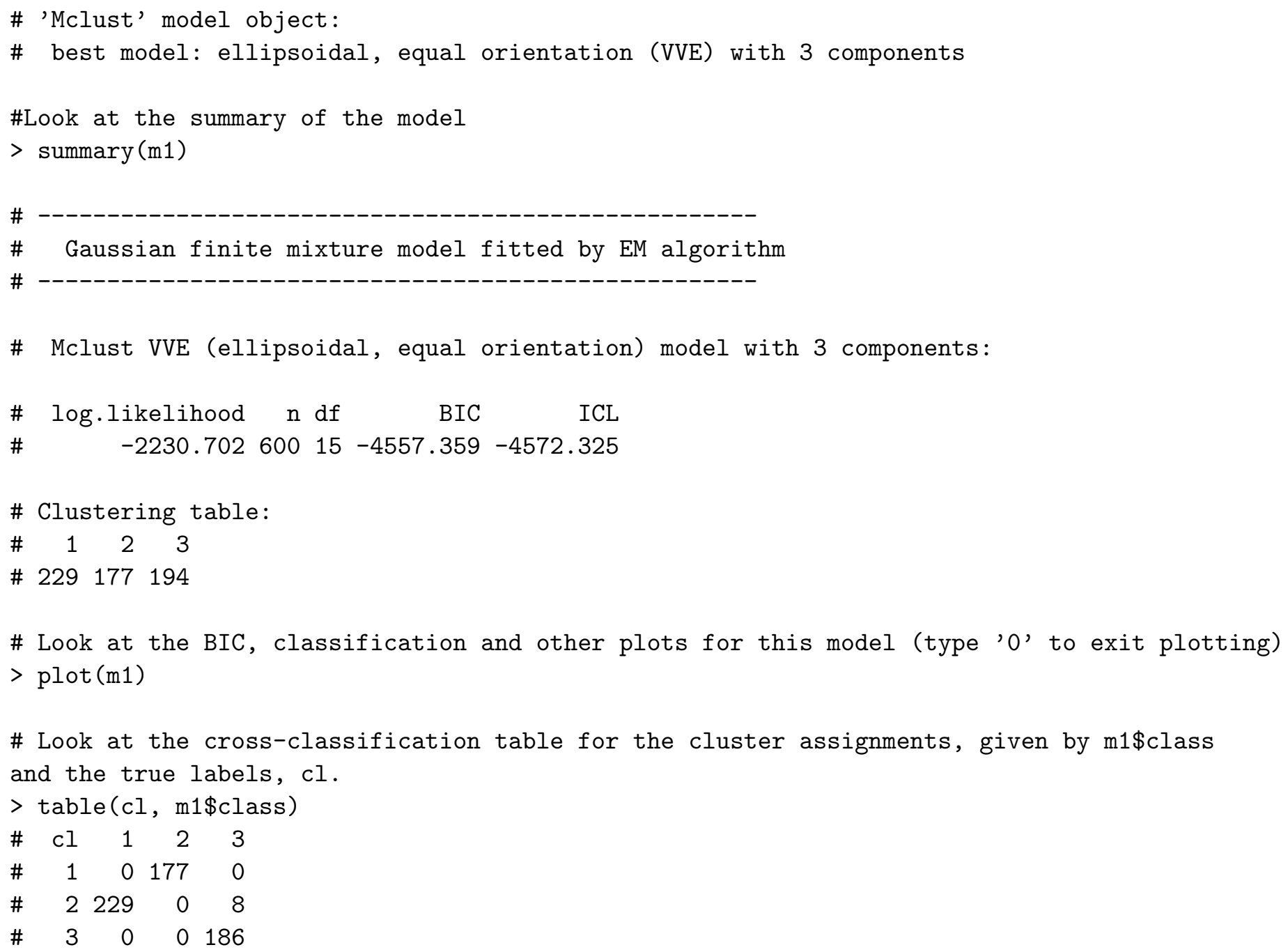

The 3-component model with variance matrix parameterization "VVE," had the highest BIC. The plot function can be used on an Mclust object to view 4 different plots: BIC (Figure 4a), classification (Figure 4b), uncertainty and density. This method correctly clusters all but 8 of the observations from the red group by assigning them to the green group (misclassification rate $=0.01 \overline{3}$ ), as seen in the cross-classification table, above.

\subsubsection{Latent Class Analysis}

Latent class analysis (LCA) is the common name for finite mixture model clustering for categorical data. LCA assumes conditional independence for the variables given component membership, i.e. all the variables are independent within the clusters (but not marginally independent) similar to the 
'XXI' models in the continuous case for model-based clustering. Each variable within each component is modeled by a multinomial variable. Each component can be summarized using the proportions for each category in each variable (as well as the mixing proportion).

On a technical note, a necessary (but not sufficient) condition for identifiability of a G component/class/cluster LCA model to be fit to data with $k$ variables, each having levels $d_{i}, i=1 \ldots, k$ is:

$$
d_{1} \times d_{2} \times \cdots \times d_{k}>\left(d_{1}+d_{2}+\ldots+d_{k}-k+1\right) \times G
$$

\subsubsection{Simulated Data Results and Code for Latent Class Analysis}

In the poLCA library (Linzer and Lewis, 2011) the poLCA command is used to fit the LCA model. Similar to kmeans, one should fit a range of consecutive numbers for nclass and choose the model with the lowest values of reported AIC or BIC scores.

The first argument in the poLCA command is a formula with the data to be clustered (variables in columns) on the left separated by a tilde from a 1 on the right e.g. data $\sim 1$. The assignment of points to their estimated class can be found in the predclass element of the fitted poLCA object (e.g.

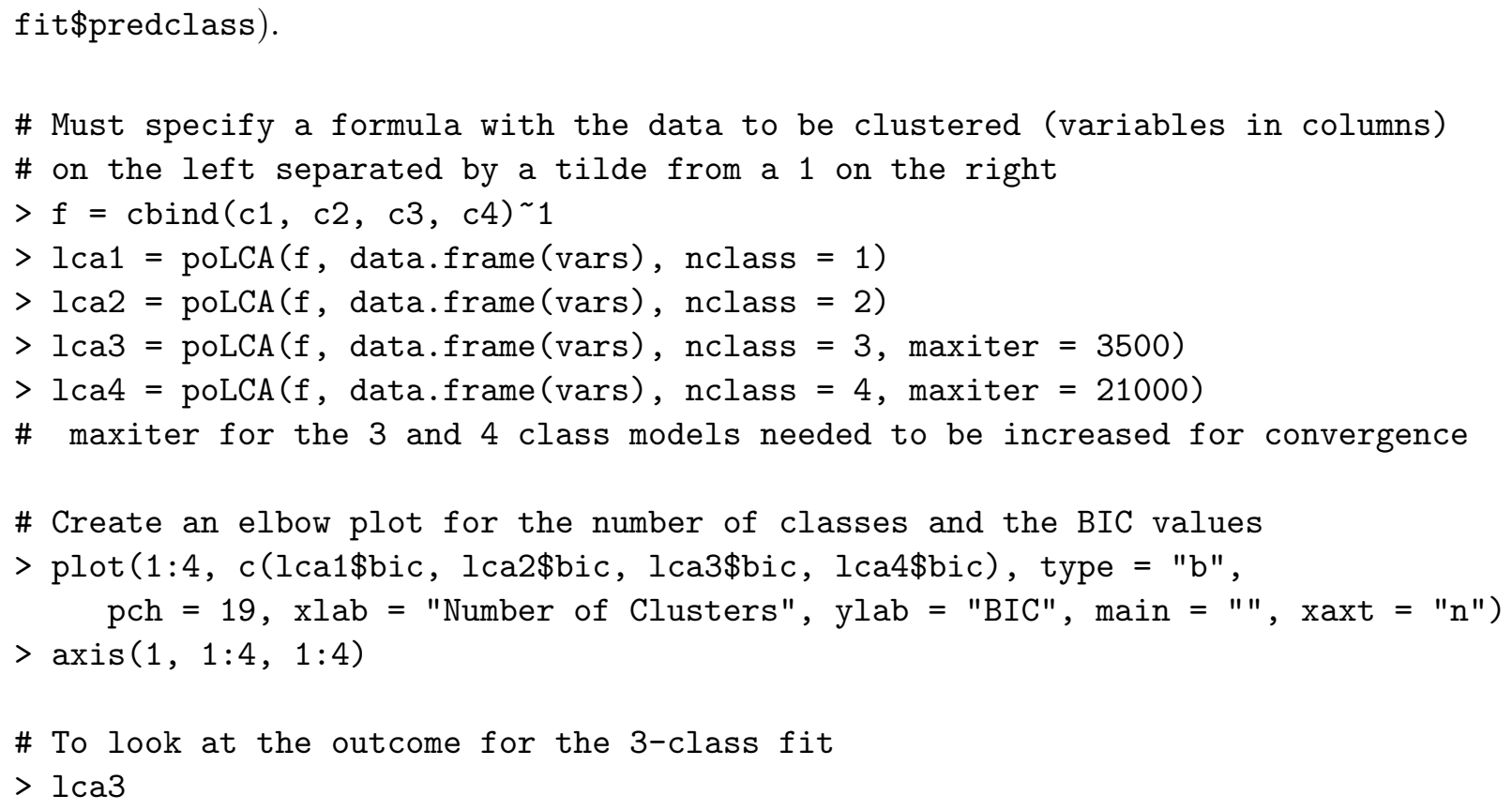




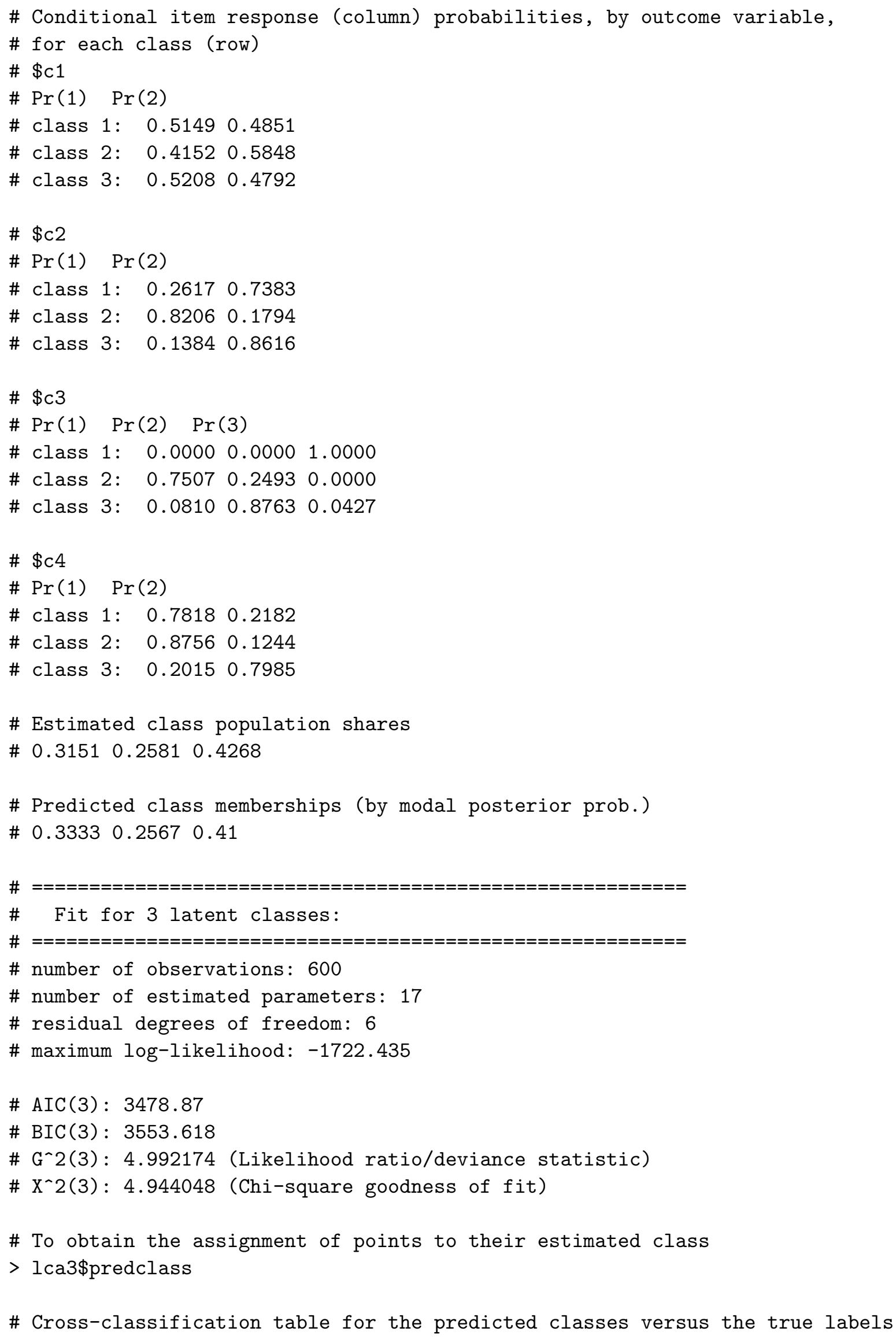


$>$ table (cl, lca3\$predclass)

$\begin{array}{lllll}\# & c l & 1 & 2 & 3\end{array}$

\# $\quad 1 \quad 34 \quad 129 \quad 14$

$\begin{array}{llllll}\# \quad 2 & 15 & 12 & 210\end{array}$

$\begin{array}{lllll}\# & 3 & 151 & 13 & 22\end{array}$

The elbow plot illustrating the BIC results for fitting an LCA model to the discrete variables for 1 to 4 clusters is given in Figure 5. Note that lower BIC is better here. This plot suggests the use of 3 clusters. Because the underlying simulated groups were less defined by the discrete variables (see Section 2.1), this model has a higher misclassification rate of $0.18 \overline{3}$.

\subsubsection{Model-Based Clustering for Mixed Data}

The clustMD library (McParland, 2015) presents an elegant model-based clustering approach to grouping sets of data with all types of data: continuous, binary, ordinal and nominal variables. Coding of categorical variables again must start at 1 and increase by unit increments. The non-continuous variables are modeled as categorizations of unseen latent Gaussian variables in the item response theory vein. However, the model-based clustering approach means that within each cluster, latent variables are modeled by their own Gaussians, different to that in other clusters. This results in a finite mixture model of both observed continuous variables and unobserved/latent Gaussian variables. As with the mclust approach, parsimony is achieved by restricting elements of the variance matrices across the clusters. (The models available are: "EII", "VII", "EEI", "VEI", "EVI" or "VVI" in the same fashion as in mclust.)

\subsubsection{Simulated Data Results and Code for Model-Based Clustering for Mixed Data}

The clustMD command's first argument is the data matrix which must order the columns with first, the continuous variables, followed by the binary, ordinal and then nominal variables (except in cases where there are nonesuch). The second argument identifies the number of components to fit, the third says how many continuous variables are in the dataset and the fourth how many total variables there 
are in the data. The argument model is a string entry indicating which of the parsimonious models is to be used.

The function clustMD is used on the full set of 6 variables, which are continuous and discrete. This mixed model is fit for a range of 1 to 4 groups and all combinations of the 6 available variance matrix parameterizations.

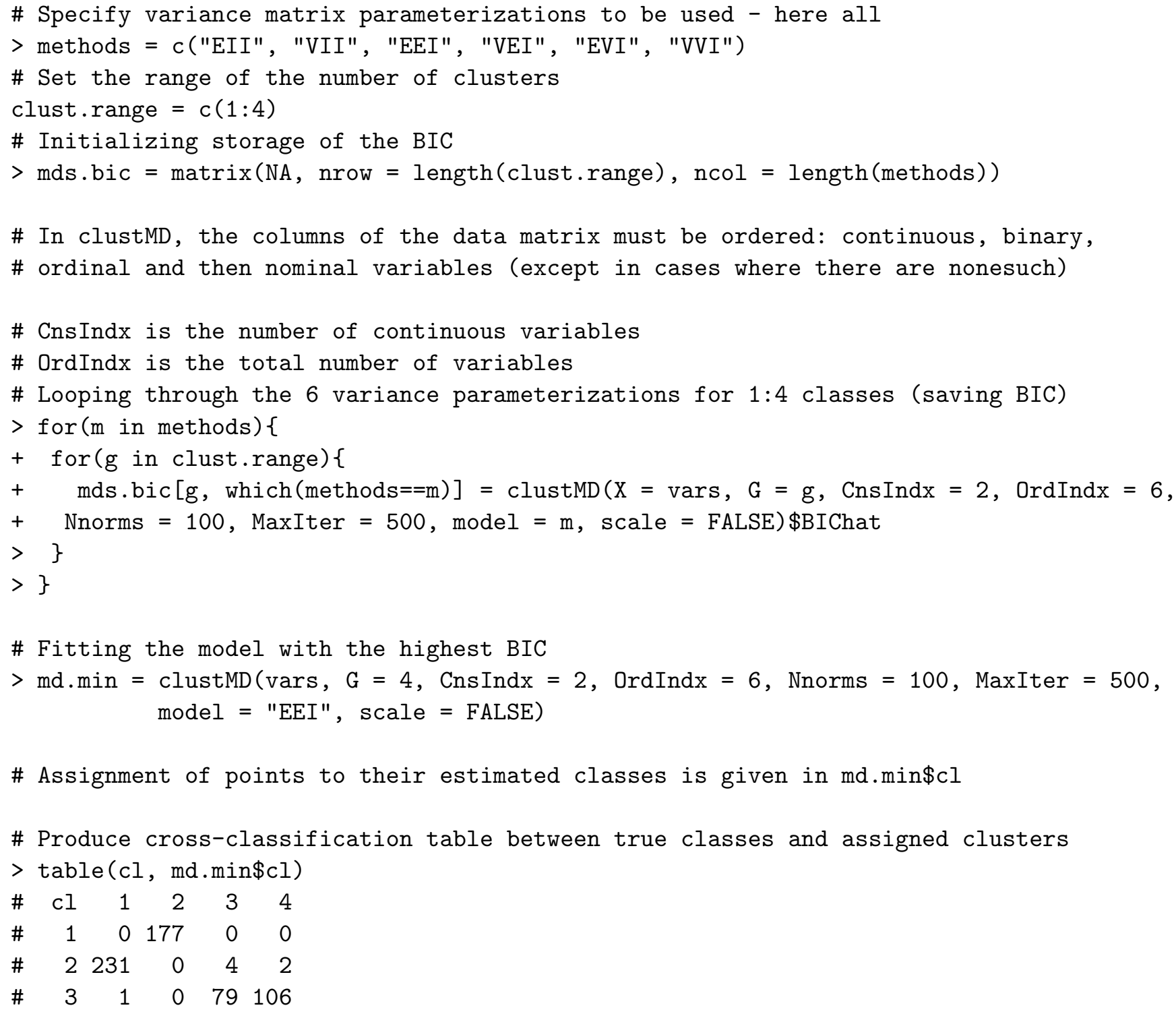

The model with the highest BIC (see Figure 6a) was the 4 cluster solution with parameterization "EEI." As seen in Figure 6b where observations are colored by their estimated cluster, this model has essentially split the original green group into two clusters and resulted in a misclassification rate of 
$0.14 \overline{3}$.

\section{Discussion}

As illustrated in Section 2, different clustering methods applied to the same data can produce vastly different results. It is difficult to recommend which method will work in practice since different group/data types will require different methods and it is seldom obvious a priori which is optimal. There is no one cluster method that is best in every situation, unfortunately. Cluster analysis as discussed in this article should be considered an exploratory analysis, and we recommend replicating/validating cluster results on one dataset with a later dataset of the same type before making any definitive statements.

In general, single linkage hierarchical clustering should really only be used for the identification of outliers because it is rarely useful otherwise. Sometimes the type of cluster solution you want should be driven by substantive reasons, rather than by the data. If on believes a genuine hierarchy of clustering solutions for the data, hierarchical clustering is then preferred. If long, elongated clusters do not make substantive sense (do you want a cluster with wide variability in one variable and not others?), then general model-based clustering models might be avoided in favor of spherical models (like "EII" and "VII" or k-means or Ward's linkage hierarchical clustering).

\subsection{Alternative Methods \& Extensions}

Although a full survey of the available extensions outside of the basic models/algorithms already introduced is beyond the scope of this article, we offer references to a few popular alternatives and extensions.

One alternative to k-means that does not use the centroid as a measure of the center of the cluster but instead uses an actual central data point is called k-medoids. This is useful when you want real 
data points as the prototypes for the clusters found. The command for implementing this in the cluster library (Maechler et al., 2015) is pam (short for partitioning around medoids).

Instead of agglomerative merging, it is also possible to have a divisive version of hierarchical clustering which starts with all observations in one cluster and successively splits clusters in two. This is not generally as popular as the agglomerative version but the command diana in the cluster package executes this algorithm.

As an alternative to model-based clustering of mixed data, one can define a distance measure on the continuous and categorical variables and apply distance methods such as hierarchical clustering (as discussed previously) if preferred. Hennig and Liao (2013) gives a good example of how to construct such a measure in a principled fashion.

There are also recent extensions to the clustering methodology presented, such as clusterwise regression (see the package flexmix (Gruen and Leisch, 2008) and poLCA for fitting such models as finite mixtures of regressions), variable selection (see clustvarsel or Rmixmod (Auder et al., 2014)); or combining mixture components to create multi-component clusters (see the clustCombi command in mclust). Also, an example of a package implementing non-parametric clustering, that we mentioned in the introduction but do not review, is pdfCluster (Azzalini and Menardi, 2014).

One comprehensive resource for cluster analysis packages on $\mathrm{R}$ is the CRAN Task View page on Cluster Analysis \& Finite Mixture Models found at http://finzi.psych.upenn.edu/views/Cluster.html. It provides short summaries of the functionalities of all the packages currently known to be available for clustering in $\mathrm{R}$ and is updated on a regular basis. Many of the packages mentioned also provide in-depth vignettes, giving more detail on their usage. Finally, the R command example is useful for automatically running the example code in help files for package commands to give an idea of how the command is to be used (e.g. example(kmeans)). 


\section{References}

Anderson, C., Lee, D., and Dean, N. (2014). Identifying clusters in Bayesian disease mapping. Biostatistics, 3(15), 457-469.

Auder, B., Lebret, R., Iovleff, S., and Langrognet, F. (2014). Rmixmod: An interface for MIXMOD. $\mathrm{R}$ package version 2.0.2.

Azzalini, A. and Menardi, G. (2014). Clustering via nonparametric density estimation: The R package pdfCluster. Journal of Statistical Software, 57(11), 1-26.

Dean, N. and Nugent, R. (2013). Clustering student skill set profiles in a unit hypercube using mixtures of multivariate betas. Advances in Data Analysis and Classification, 3(7), 339-357.

Dempster, A. P., Laird, N. M., and Rubin, D. B. (1977). Maximum likelihood from incomplete data via the EM algorithm. Journal of the Royal Statistical Society. Series B (Methodological), 39(1), $1-38$.

Everitt, B., Landau, S., Leese, M., and Stahl, D. (2011). Cluster Analysis. Wiley Series in Probability and Statistics. Chichester, UK: Wiley.

Flynt, A. and Daepp, M. I. (2015). Diet-related chronic disease in the northeaster united states: a model-based clustering approach. International Journal of Health Geographics, pages 14-25.

Fraley, C. and Raftery, A. E. (2002). Model-based clustering, discriminant analysis, and density estimation. Journal of the American Statistical Association, 97(458), 611-631.

Fraley, C., Raftery, A. E., Murphy, T. B., and Scrucca, L. (2012). mclust version 4 for r: Normal mixture modeling for model-based clustering, classification, and density estimation. Technical Report 597, Department of Statistics, University of Washington. 
Gower, J. C. and Ross, G. J. S. (1969). Minimal spanning tree and single linkage cluster analysis. Journal of the Royal Statistical Society, Series C (Applied Statistics), 18(1), 54-65.

Gruen, B. and Leisch, F. (2008). Flexmix version 2: Finite mixtures with concomitant variables and varying and constant parameters. Journal of Statistical Software, 28(4), 1-35.

Hennig, C. (2015). fpc: Flexible Procedures for Clustering. R package version 2.1-10.

Hennig, C. and Liao, T. F. (2013). How to find an appropriate clustering for mixed type variables with application to socioeconomic stratification. Journal of the Royal Statistical Society, Series C (Applied Statistics), 62(3), 309-369.

Lazarsfeld, P. F. (1950a). Measurement and Prediction, volume IV of The American Soldier: Studies in Social Psychology in World War II, chapter The Logical and Mathematical Foundations of Latent Structure Analysis, pages 362-412. Princeton University Press.

Lazarsfeld, P. F. (1950b). Measurement and Prediction, volume IV of The American Soldier: Studies in Social Psychology in World War II, chapter Some Latent Structures. Princeton University Press.

Lazarsfeld, P. F. and Henry, N. W. (1968). Latent Structure Analysis. Boston: Houghton Mifflin.

Linzer, D. A. and Lewis, J. B. (2011). poLCA: An R package for polytomous variable latent class analysis. Journal of Statistical Software, 42(10), 1-29.

MacQueen, J. (1967). Some methods for classification and analysis of multivariate observations. In Proceedings of the Fifth Berkeley Symposium on Mathematical Statistics and Probability, Volume 1: Statistics, pages 281-297, Berkeley, Calif. University of California Press.

Maechler, M., Rousseeuw, P., Struyf, A., Hubert, M., and Hornik, K. (2015). cluster: Cluster Analysis Basics and Extensions. R package version 2.0.3.

McLachlan, G. J. and Peel, D. (2000). Finite Mixture Models. New York: Wiley. 
McParland, D. (2015). clustMD: Model Based Clustering for Mixed Data. R package version 1.1.

R Core Team (2015). R: A Language and Environment for Statistical Computing. R Foundation for Statistical Computing, Vienna, Austria.

Schwarz, G. E. (1978). Estimating the dimension of a model. Annals of Statistics, 6(2), 461-464.

Ward, J. H. (1963). Hierarchical grouping to optimize an objective function. Journal of the American Statistical Association, 58(301), 236-244.

Wolfe, J. H. (1963). Object cluster analysis of social areas. Master's thesis, University of California, Berkeley. 


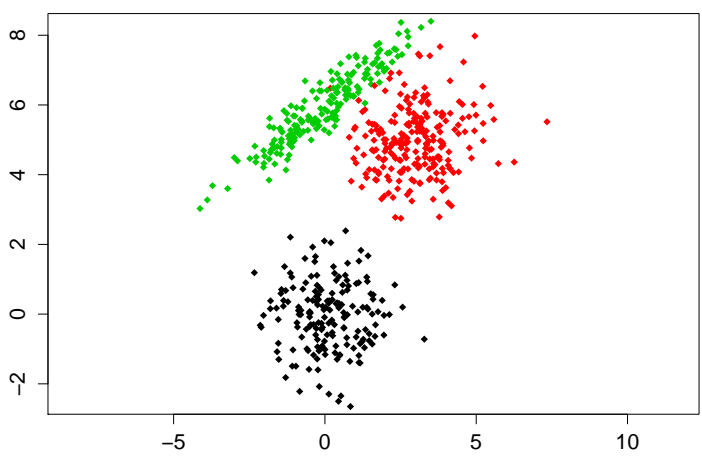

(a) Continuous variables, colored by 'true' class assignment

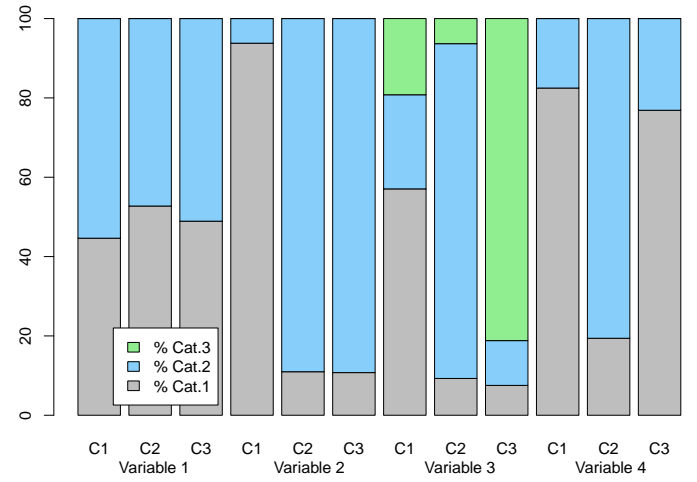

(b) Discrete variables, where a single bar is labeled by each cluster and variable combination

Figure 1: Simulated Data Set 


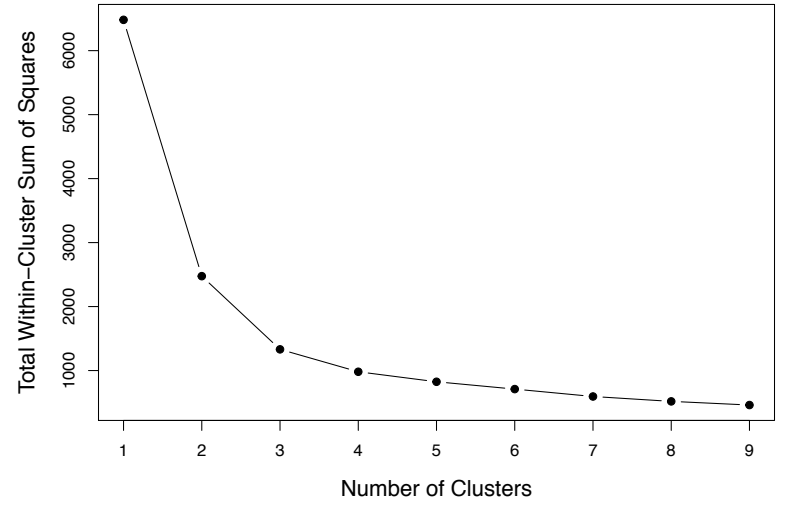

Figure 2: Elbow plot for the total within-cluster sum of squares from k-means models 


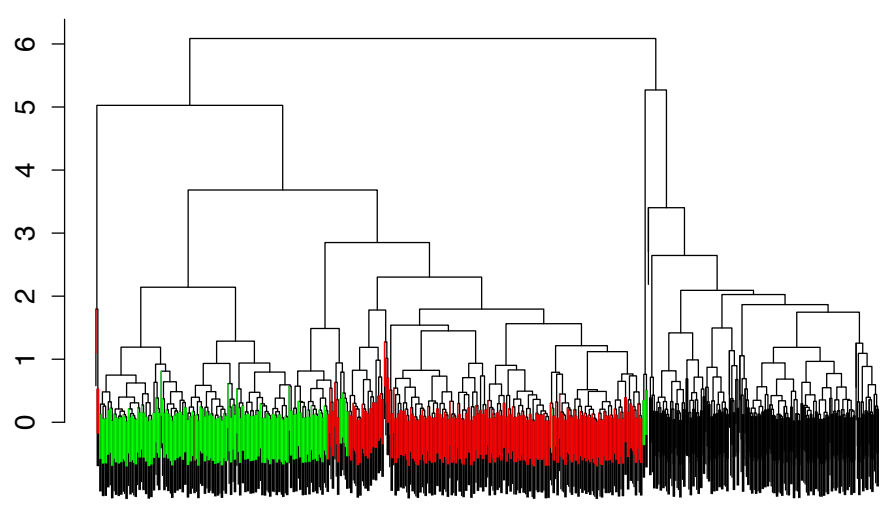

Figure 3: Colored dendrogram resulting from hierarchical clustering using average linkage 


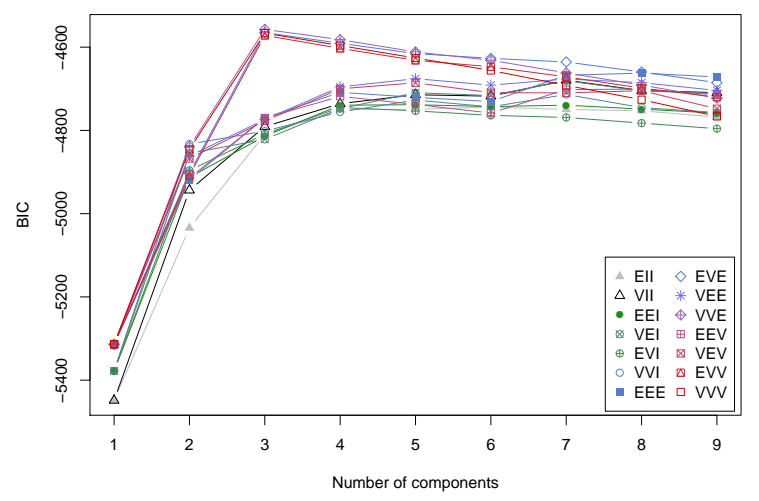

(a) BIC results for all 12 variance matrix parameterizations and possible clusters 1 to 9

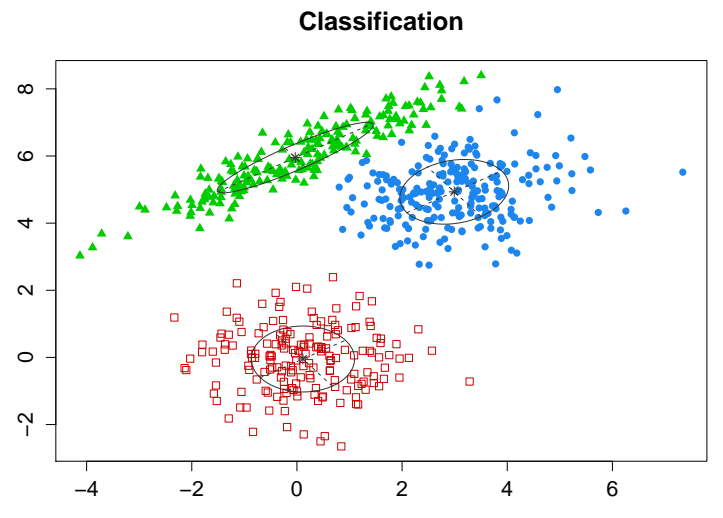

(b) Classification results for the 3 component solution

Figure 4: Two example plots produced by Mclust 


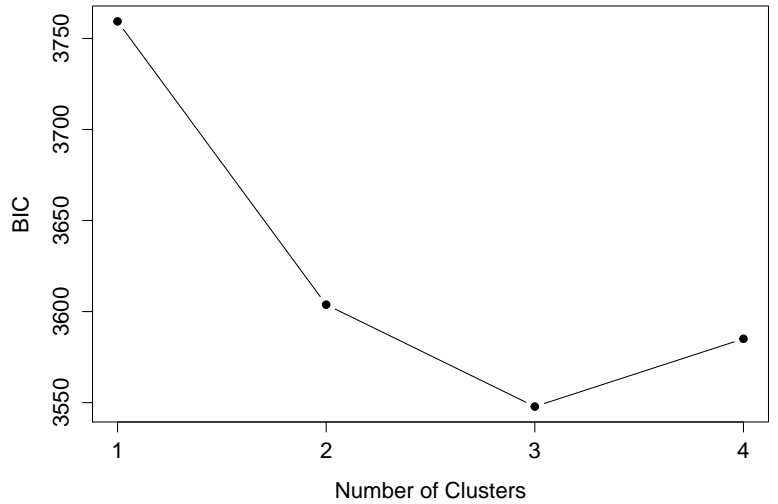

Figure 5: Elbow plot for poLCA models fit on 1 to 4 clusters 


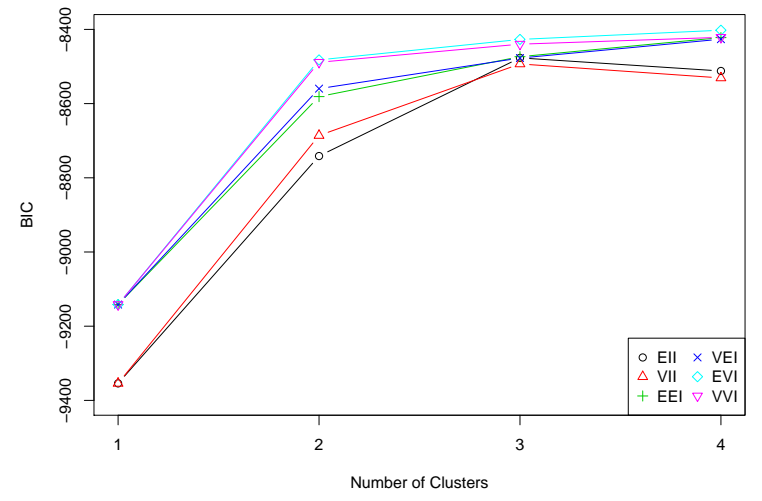

(a) BIC values for models fit on 1 to 4 clusters for the 6 available variance matrix parameterizations

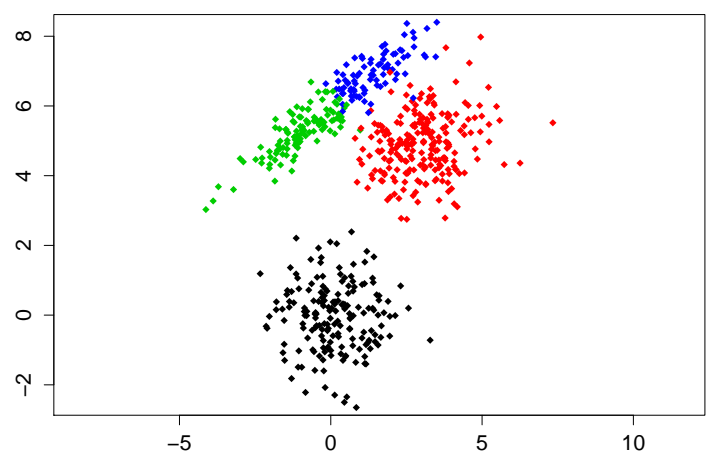

(b) Clustering solution from the best chosen mixed data model

Figure 6: Results for clustMD models 